Article

\title{
An Intelligent Quadrotor Fault Diagnosis Method Based on Novel Deep Residual Shrinkage Network
}

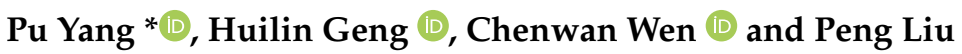 \\ Department of Automation, Nanjing University of Aeronautics and Astronautics, Nanjing 210016, China; \\ hlgeng@nuaa.edu.cn (H.G.); wenchenwan@nuaa.edu.cn (C.W.); llpeng@nuaa.edu.cn (P.L.) \\ * Correspondence: ppyang@nuaa.edu.cn
}

Citation: Yang, P.; Geng, H.; Wen, C.; Liu, P. An Intelligent Quadrotor Fault Diagnosis Method Based on Novel Deep Residual Shrinkage Network. Drones 2021, 5, 133. https://doi.org/ $10.3390 /$ drones5040133

Academic Editor: Marija Popović

Received: 8 October 2021

Accepted: 4 November 2021

Published: 8 November 2021

Publisher's Note: MDPI stays neutral with regard to jurisdictional claims in published maps and institutional affiliations.

Copyright: (c) 2021 by the authors. Licensee MDPI, Basel, Switzerland. This article is an open access article distributed under the terms and conditions of the Creative Commons Attribution (CC BY) license (https:// creativecommons.org/licenses/by/ $4.0 /)$.

\begin{abstract}
In this paper, a fault diagnosis algorithm named improved one-dimensional deep residual shrinkage network with a wide convolutional layer (1D-WIDRSN) is proposed for quadrotor propellers with minor damage, which can effectively identify the fault classes of quadrotor under interference information, and without additional denoising procedures. In a word, that fault diagnosis algorithm can locate and diagnose the early minor faults of the quadrotor based on the flight data, so that the quadrotor can be repaired before serious faults occur, so as to prolong the service life of quadrotor. First, the sliding window method is used to expand the number of samples. Then, a novel progressive semi-soft threshold is proposed to replace the soft threshold in the deep residual shrinkage network (DRSN), so the noise of signal features can be eliminated more effectively. Finally, based on the deep residual shrinkage network, the wide convolution layer and DroupBlock method are introduced to further enhance the anti-noise and over-fitting ability of the model, thus the model can effectively extract fault features and classify faults. Experimental results show that 1D-WIDRSN applied to the minimal fault diagnosis model of quadrotor propellers can accurately identify the fault category in the interference information, and the diagnosis accuracy is over $98 \%$.
\end{abstract}

Keywords: quadrotor; minor fault diagnosis; network design process pattern; 1D-WIDRSN

\section{Introduction}

UAV is a reusable unmanned aerial vehicle controlled by remote control or its own procedures through radio technology. After entering the 21st century, UAV has shown its skill in various fields. In the Marine industry, UAV can be used to complete coastal zone monitoring and Marine mineral exploration [1]. In agriculture, UAV can be used to fertilize fields and monitor the growth status of crops [2]. In geology, UAV can be used to conduct geological disaster investigation and geological mapping [3]. In terms of traffic management, UAV can be used to monitor road conditions and assist in dealing with traffic accidents [4].

As a representative of small multi-rotor aircraft, quadrotor UAV has the advantages of small size, light weight, and easy control, so it is widely favored by people. The quadrotor has four rotors that generate upward thrust and torque, which control the speed of the rotator to perform various actions [5]. With the popularization of quadrotor UAV, the research on its fault identification and diagnosis has become more and more important. Under the long-term operation of the quadrotor, its actuator system, namely the electronic governor, motor and propellers, bears a heavier load. If the actuator system has a serious failure, such as serious damage to the propeller or serious failure of the motor, the UAV will not be able to obtain the required lift, resulting in flight instability or even crashing, which will eventually cause serious property losses and even casualties.

If there is a minor fault in the actuator of the UAV, the output power of the UAV will be reduced correspondingly. In this case, the UAV can still fly, but its stability and attitude will be slightly different from the healthy UAV. Although the quadrotor has good fault-tolerant 
control capabilities, it is also necessary to diagnose the fault when it is in the early state, because the accumulation of such minor faults may eventually cause serious problems such as the crash of UAVs. So it is of great significance to find a method to accurately detect and locate the early minor faults of UAV. However, due to the interference of the external environment and the fault-tolerant control of the UAV itself, these potential faults such as minor faults of actuator are difficult to be detected. Therefore, how to accurately diagnose the early minor faults of the UAV actuator from the numerous interference information is an urgent problem to be solved.

Especially for UAVs used for disaster surveys, such UAVs need to fly into various unknown dangerous locations, such as areas suffering from earthquakes, floods, and tornadoes. This kind of UAV is more likely to suffer body damage caused by external collisions, so the propellers and motors of such UAVs are more prone to failure. Therefore, designing a fault diagnosis method, which can diagnose minor faults in time when the propeller of the UAV is damaged in the early stage, is of great significance to the practical application of UAV.

In recent years, many scholars have used model-based methods to identify the actuator failures of quad-rotator UAV, such as Zhang et al. [6] used interactive multi-models to detect and locate single-actuator failures of quad-rotors. Avram et al. [7] used a non-linear adaptive estimation method to detect and isolate quad-rotor actuator failures. Bauer et al. [8] used a multi-model adaptive estimation method for fault detection and reconstruction of small UAVs. Miao et al. [9] designed and constructed a UAV fault diagnosis algorithm based on adjustable nonlinear PI observer to realize the effective fault identification of continuous-time system. Kim et al. [10] used the extended Kalman filter to estimate the efficiency parameters of each UAV actuator, and the estimated coefficients were used to perform fault diagnosis. Antonio et al. [11] developed a robust actuator fault diagnosis algorithm based on Adaptive eXogenous Kalman Filter by combining nonlinear observer and linearized adaptive Kalman filter, and the algorithm tested for actuator fault diagnosis of a hexacopter UAV. The model-based diagnosis method has good robustness and can diagnose unknown faults, but the premise of this method is to establish an accurate model for the target system, but the structural parameters of the quadrotor UAV are relatively complicated. Therefore, this method relies too much on the mathematical model of the object, which is not easy to implement in practical applications and has limited scalability.

Others uesd signal processing-based methods to detect faults. This kind of method does not need to establish a quantitative or qualitative mathematical model of the system, but only collects flight data and extracts fault features from the original data through signal processing to diagnose system faults. For example, Rangel-Magdaleno et al. [12] use discrete wavelet transform and Fourier transform to process audio data to identify propeller unbalance faults of unmanned aerial vehicles. Yousefi et al. [13] used logistic regression and linear discriminant analysis algorithm to detect unmanned aerial vehicle faults after de-noising the voltage and current data of the four actuators of the UAV. Park et al. [14] used multivariate statistical analysis techniques such as partial least squares regression to process data and diagnose propeller failure. Bondyra et al. [15] used wavelet packet decomposition, fast Fourier transform, and signal power spectrum to extract features of vibration signals under single-sided damage of the UAV propellers, and then advanced fault diagnosis through support vector machine classifier. Altinors et al. [16] applied Decision Tree, Support Vector Machines, and K Nearest Neighbor algorithms to machine learning, and applies these algorithms to the sound data received by the motor for UAV fault diagnosis.

With the development of computational intelligence, deep learning has become a hot spot in the field of fault detection. Fault diagnosis method based on neural network preprocesses the original data and inputs it into the neural network model to obtain the fault diagnosis result directly. Chen et al. [17] first used wavelet packets to extract energy entropy, and then used a genetic algorithm optimized BP neural network to construct a fault diagnosis model to realize the detection of UAV sensor faults. Guo et al. [18] used short- 
time Fourier changes to transform the signal into a time-frequency graph, and then used a convolutional neural network to extract fault features to realize the sensor fault diagnosis of the UAV. Iannace et al. [19] collected the audio on the UAV propellers in flight, and used the artificial neural network model to analyze it to detect faults. Gao et al. [20] used the raw data of MEMS inertial sensors as input to establish a multi-scale convolutional neural network model to diagnose the fault of the UAV temperature sensor. Xiao [21] used wavelet packet to extract the fault characteristics of UAV sensors, and proposed a observer method based on BP neural network to detect and process single or multiple sensor faults online. Liu et al. [22] transformed the audio data of UAV flight into time-frequency spectrogram for diagnosis, and developed a diagnostic model based on convolutional neural network and transfer learning techniques, realizing the damage detection of propeller. Jia et al. [23] proposed a novel deep network constructed by multi-layer extreme learning machine based auto-encoder to solve the problem of UAV actuator fault diagnosis under imbalanced data, the deep network has the advantages of strong feature mining ability, high accuracy and fast speed.

It can be seen that the research on the fault diagnosis of quad-rotor UAVs based on data-driven has attracted more and more attention. This method is widely used and computationally efficient, taking into account the uncertainty of the model and the sensor noise. However, in previous studies, most of the fault diagnosis methods of quadrotor UAV need to extract fault features through some signal processing methods before fault diagnosis, and cannot directly realize the fault diagnosis of UAV through the original signal. Therefore, end-to-end fault diagnosis based on convolutional neural networks still has a lot of research space. Among them, one-dimensional convolution neural network is often used in sequence model, which can analyze the time series of sensor data (such as gyroscope and acceleration meter data), and realize self-extraction of fault features in time domain $[24,25]$. It integrates feature extraction, feature dimension reduction and pattern recognition to achieve end-to-end fault diagnosis.

In previous studies, research on quadrotor fault diagnosis is mostly aimed at obvious faults. In this case, quadrotor has shown an obvious fault phenomenon, and the relevant research has been relatively mature. However, there are few studies on the early minor faults of the quadrotor actuator system, and most of the methods currently have low diagnostic accuracy. In view of the above problem, this paper mainly focuses on the early minor faults of the actuator when the quadrotor can still fly, and tries to diagnose and locate these faults, so as to avoid serious accidents caused by the accumulation of minor faults.

The propeller minor damage fault is one of the common fault types of quadrotor. In addition, different fault locations have different effects on the quadrotor. To solve the problem of low accuracy of propeller minor damage fault diagnosis for quadrotor caused by the multiple interference information and the difficulty in feature extraction of quadrotor, this paper takes a certain type of quadrotor used for disaster investigation as the object, proposed a diagnosis algorithm based on 1D deep residual shrinkage network with a wide convolution layer (1D-WIDRSN). The algorithm integrates feature extraction, feature dimension reduction and pattern recognition to achieve end-to-end fault diagnosis. The innovative contributions of this paper are summarized as follows:

(1) An end-to-end fault diagnosis method is presented. The original flight data containing disturbance information can be directly input into the fault diagnosis model to obtain the diagnosis result. The fault diagnosis model based on residual shrinkage network integrates soft threshold and attention mechanism, which can realize the fault diagnosis of quadrotor under the influence of external environment interference and UAV fault tolerant control.

(2) In order to retain more fault characteristic information, we improved the traditional residual shrinkage network: a new shrinkage function named progressive semi-soft threshold function is proposed to replace the original soft threshold function.The traditional soft threshold will eliminate the effective data features beyond the interference information, and the new progressive semi-soft threshold can retain the 
continuity of the soft threshold and eliminate the signal distortion caused by the soft threshold, which further improves the diagnostic accuracy of the model.

(3) In order to enhance the feature learning ability of the model, a suitable wide convolutional layer is introduced as the first layer of the fault diagnosis model. The wide convolutional layer can effectively extract the short-term features of the original flight data, and further improve the feature extraction capability of the fault diagnosis model.

(4) In order to prevent the model from over-fitting, the DropBlock layer is introduced when training the model. By randomly discarding part of the feature blocks, the model can be extracted several times to get the optimal features, which improves the training speed and anti-over-fitting ability of the fault diagnosis model.

The rest of this paper is organized as follows: Section 2 describes the construction process, modeling algorithm and optimization strategy of 1D-WIDRSN model. Section 3 explains the data acquisition, related the model structure determination experiment. Section 4 introduces the evaluation and discussion of model performance. Section 5 presents the conclusion and future work directions.

\section{Proposed Methods}

\subsection{Overview}

To confront the challenge of fault diagnosis caused by minor fault, a fault diagnosis model based on 1D-WIDRSN is proposed. This section introduces the design principles and architecture of the 1D-DRSN in detail. The framework of 1D-WIDRSN model is shown in Figure 1, which consists of a wide convolution layer and multiple residual shrinkage modules.

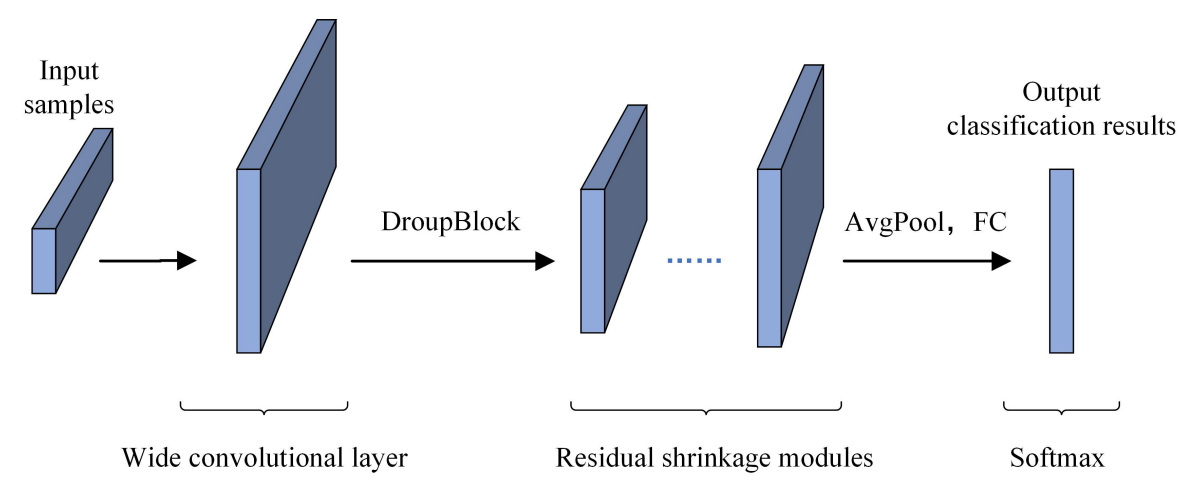

Figure 1. Framework of the fault diagnosis model based on 1D-WIDRSN.

\subsection{Deep Residual Shrinkage Network (DRSN) Model}

In order to solve the degradation problem of multilayer convolutional neural network model, He et al. [26] proposed a residual network, which is composed of a series of residual blocks. It is an improved convolutional neural network. By introducing cross layer links, the residual network can still learn new features based on the input features, thereby solving the degradation problem caused by the increase of network depth. The residual block is shown as follows:

$$
a^{l+1}=a^{l}+F\left(a^{l}, W^{l}\right)
$$

where $a^{l}$ represents the input of the $l$-th residual module, $F$ represents the residual function.

Zhao et al. [27] proposed the concept of deep residual contraction network (DRSN) in 2020. DRSN is an improved ResNet by integrating soft threshold function and attention mechanism into residual network. Its working principle is to automatically find the interference features of the input samples with the help of the attention mechanism, and set them to zero through the soft threshold function, so as to realize the ability of extracting data features from the interference information. 
The feature selection process of deep residual shrinkage network can be understood as follows: Firstly, through convolution layer and pooling layer, important information such as fault features are converted to larger absolute values, and irrelevant features corresponding to interference information such as noise are converted to smaller absolute values. Then, the boundary between the two is obtained through the attention mechanism, and the interference information is zeroed by soft thresholding. Finally, only the important information is output. Deep residual shrinkage module (DRSM) as shown in Figure 2.

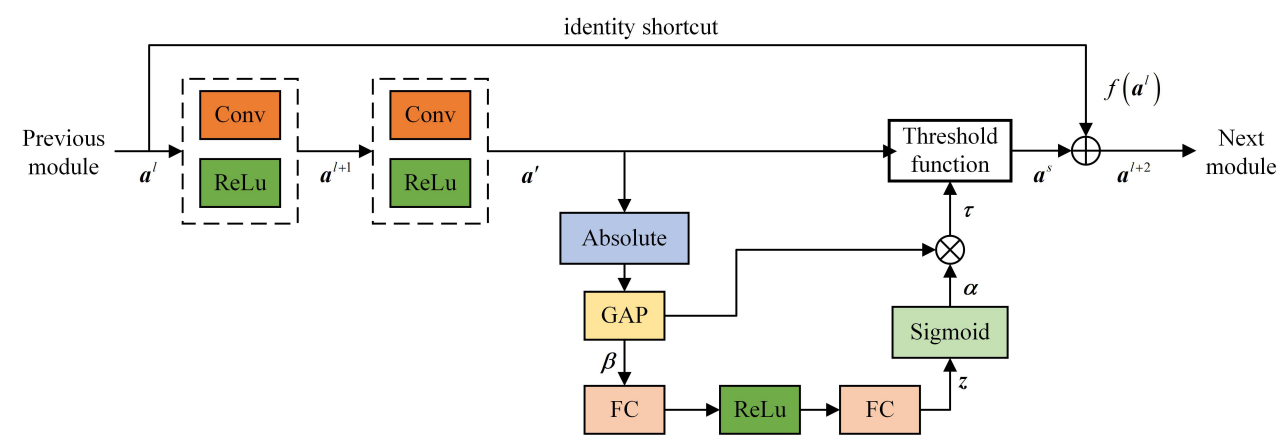

Figure 2. Deep residual shrinkage module.

$a^{l}$ is the input of the module. After passes through the first convolution layer, $a^{l+1}$ is obtained through ReLU function, and then $a^{l+1}$ is input into the second convolution layer. The second convolution layer constructs a sub grid to obtain the threshold. Then, the output $a^{\prime}$ of the second convolution layer is quantized by taking the absolute value and global average pooling (GAP) to obtain a one-dimensional vector $\beta, \beta$ represents the mean parameter, then the characteristics of the channel are mined through two full connection layers, and finally the attention weight $z$ is obtained through the activation function Sigmoid, Each attention weight parameter $\alpha \in(0,1)$ acts on the feature vector of the corresponding feature channel. $\alpha$ and $\beta$ are multiplied to obtain the threshold $\tau$, so each characteristic channel has an independent threshold. Finally, the obtained threshold $\tau$ is used for soft threshold processing to obtain $a^{s}, a^{s}$ is added to the residual term $f\left(a^{l}\right)$ to obtain module output $a^{l+2}$. The calculation formula of the specific process is as follows:

$$
\begin{gathered}
a^{l+1}=\operatorname{ReLu}\left(w^{l+1} a^{l}+b^{l+1}\right) \\
a^{\prime}=\operatorname{ReLu}\left(w^{l+2} a^{l+1}+b^{l+2}\right) \\
\beta=\text { average }\left|a^{\prime}\right| \\
\alpha=\frac{1}{1+e^{-z}} \\
\tau=\alpha \cdot \beta \\
a^{s}=\left\{\begin{array}{cc}
\operatorname{sgn}\left(a^{\prime}\right) \cdot\left(\left|a^{\prime}\right|-\tau\right) & \left|a^{\prime}\right| \geq \tau \\
0 & \left|a^{\prime}\right|<\tau
\end{array}\right. \\
a^{l+2}=a^{s}+f\left(a^{l}\right)
\end{gathered}
$$

where, $w^{l+1}$ and $w^{l+2}$ is the weight of $l+1$ layer and $l+2$ layer, $b^{l+1}$ and $b^{l+2}$ is the bias of $l+1$ layer and $l+2$ layer.

\subsection{Progressive Semi-Soft Threshold}

The soft threshold in the depth residual shrinkage network realizes the denoising of the input data by setting the interference information to zero. Soft threshold denoising is a simple and traditional denoising technology. The data processed by the soft threshold function has good continuity, but when $\left|a^{\prime}\right|>\tau$, there is always a constant deviation between $a^{s}$ and $a^{\prime}$, so the soft threshold may eliminate some effective features, cause a certain degree of distortion to the data and reduce the accuracy of diagnosis [28]. 
In response to the above defects, this paper introduces a progressive semi-soft threshold function. Compared with the soft threshold function, the progressive semi-soft threshold function not only retains the continuity of the soft threshold function, but also eliminates the distortion caused by the soft threshold [29]. The calculation formula for realizing progressive semi-soft threshold is as follows:

$$
a^{s}=\left\{\begin{array}{cl}
\operatorname{sgn}\left(a^{\prime}\right) \cdot\left(\left|a^{\prime}\right|-\frac{0.5 \tau}{e^{\left|a^{\prime}\right|-\tau}-0.5}\right) & \left|a^{\prime}\right| \geq \tau \\
0 & \left|a^{\prime}\right|<\tau
\end{array}\right.
$$

The progressive semi-soft threshold process is shown in Figure 3. As shown in Figure 3, the progressive semi-soft threshold function is continuous, and there is little constant deviation between the processed signal and the real signal, which retains the effective features in the original data as much as possible.

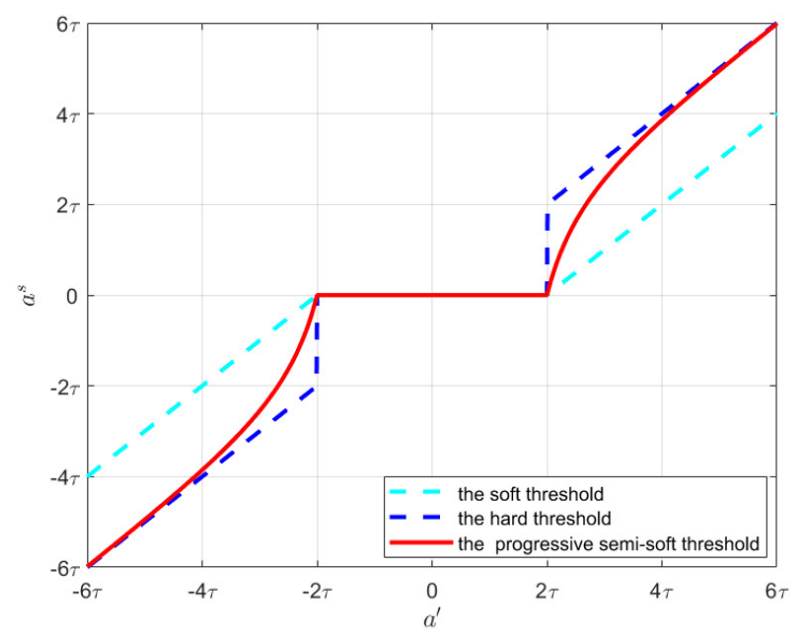

Figure 3. The function image of the new threshold function.

\subsection{Wide Convolution Layer -Enhancement of Feature Learning Ability}

In this paper, the wide convolution layer is taken as the first layer of the 1D-WIDRSN, that is, the first convolution layer of the model is set as the wide convolution kernels, and the other layers are set as the small convolution kernels. The wide convolution kernels in the first layer can better suppress the interference information, expand the receptive field area, extract short-term features, adaptively learn the important features of fault diagnosis objects and speed up model training. The small convolution kernels of other layers can reduce the network parameters, deepen the number of network layers and suppress overfitting $[30,31]$. Therefore, the configuration of the first layer wide convolution kernels and the other layers small convolution kernels can better improve the network performance and training speed.

\subsection{DropBlock Module -Enhancement of Anti-Overfitting Ability}

Ghiasi et al. [32] proposed the DropBlock regularization module for convolutional neural network. The traditional Dropout regularization method randomly discards some independent neurons to reduce the number of intermediate features, but the convolution layer will still learn interference features through the interaction between features. Therefore, Ghiasi believes that the effect of applying Dropout method in convolution layer is plain. However, by setting the block size, the DropBlock module will randomly set a block area of the feature to zero, so that the model can learn the features of other areas, so as to realize the regularization of the convolution layer and improve the robustness and anti-overfitting ability of the model. When the area size is 1 , the DropBlock method is the traditional Dropout method. In this paper, a DropBlock module is added after the wide 
convolution layer, which cannot only improve the anti-over-fitting ability, but also improve the generalization ability of the model.

\subsection{Fault Diagnosis Process}

The 1D-WIDRSN model in this paper is introduced for adaptive feature learning, which can extract key features from interference information and accurately identify fault location of quadrotor. The fault diagnosis process of 1D-WIDRSN model is shown in Figure 4, and the specific steps of the fault diagnosis process are as follows:

(1) Collect the data of UAV in normal state and fault state. After preprocessing and data enhancement, the original data is randomly divided into training sample set and test sample set, accounting for $70 \%$ and $30 \%$, respectively.

(2) Bring the training set into the constructed neural network model, set the training parameters such as iteration times and learning rate, use the back propagation algorithm to adjust the network parameters until the requirements of iteration times are met.

(3) Input the test sample set into the model to obtain the fault diagnosis results, and measure the effectiveness of the model through the fault classification accuracy. If the expected standard is reached, stop the training, otherwise readjust the parameters.

(4) Save the trained model parameters, collect flight data for fault diagnosis.

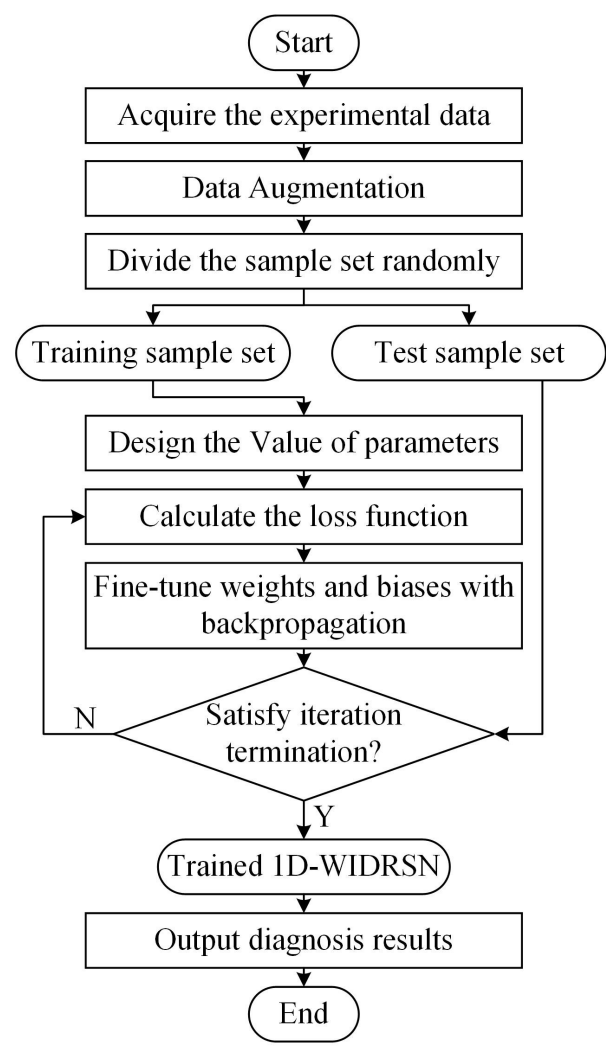

Figure 4. Flowchart of the proposed fault diagnosis model.

The training part of the 1D-WIDRSN model will be performed offline due to the need to label data, but the diagnosis part can be performed online. During the actual mission of the quadrotor, the accuracy of 1D-WIDRSN diagnosis model will be affected if environmental factors (such as excessive wind) are enough to change the attitude of the quadrotor. Therefore, in order to ensure the accuracy of the model and avoid increasing online computational cost, this fault diagnosis model is suitable for the flight-test inspection of quadrotor before or after use. 


\section{Experiments and Results}

\subsection{Data Acquisition}

In order to verify the fault detection method proposed above, it is necessary to collect actual UAV flight data for verification experiments. This part is aimed at a certain type of quadrotor, by destroying the propellers to reduce the output power of the UAV, so as to obtain the experimental data under different fault conditions. The experimental platform is mainly composed self-assembled quadrotor, computer and several propellers damaged in different degrees.

The UAV used in the experiments is an intelligent inspection quadrotor based on Pixhawk system assembled by our national science key laboratory. As shown in Figure 5, the power system of the quadrotor consists of T-Motor 2216 motor, 20 A four-in-one electronic speed controller, propellers and battery. There are four propulsion units on the quadrotor frame, and two coaxial rotor-motor pairs on each arm. Pixhawk open-source flight control system is adopted in the flight control system. The hardware structure of Pixhawk mainly includes gyroscope, accelerometer, geomagnetic module, barometer and GPS module. The software structure of Pixhawk is mainly divided into 4 layers, the top layer is the application API, and this layer is used by developers. The second layer is the application framework, under which basic flight control nodes can be operated. The third layer is the relevant library layer, which provides functions that interact with system libraries. The last layer is the operating system (Linux and RTOS), which provides hardware drivers.

In this paper, by artificially destroying the propeller in a small area, the output power of the corresponding motor will change, and then the flight attitude of the whole UAV will also change accordingly, thereby simulating the minor failure of the UAV's actuator. The normal propeller and damaged propeller are shown in Figure 6, The length of normal propeller is about $26 \mathrm{~cm}$, and the length of damaged propeller is about $23 \mathrm{~cm}$ and its end has been cut off by $3 \mathrm{~cm}$ irregularly. Experiments have verified that the failure simulation program has a certain and small impact on the flight stability of the UAV, which belongs to the category of minor failure of UAV.

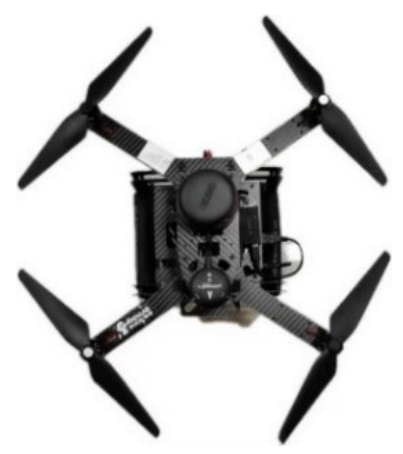

Figure 5. Quadrotor used for experimental test.

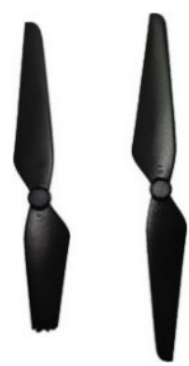

Figure 6. Propellers used for experimental test.

Five different propeller configurations are set up as shown in Table 1, including one group of normal condition and four groups of single propeller faults with the $i$-th $(i=1,2,3,4)$ 
propeller damaged. In the experiment, after a short preparation time on the ground, the aircraft is launched and immediately started its flight with a horizontal rectangular flight pattern. Figure 7 recorded the flight trajectory of the UAV in the healthy state and fault state in the experiments. It can be seen from Figure 7 that when there is a minor fault, it will be difficult to identify only through the flight trajectory curve. However, potential faults like this should not be neglected. Therefore, it is necessary to propose an effective fault detection method. In each group of experiments, the pitch rate, roll rate, yaw rate, pitch angle and roll angle of UAV in level flight are collected and saved. The yaw angle is greatly affected by manual operation, so it is not used as fault information in this paper. In actual flight, affected by external interference factors such as airspace environment and noise, the above parameters alone cannot reflect the failure of the UAV, so the output speeds Out1 Out 4 of four motors are introduced. Finally, the values of the above nine parameters are taken as the characteristic elements to characterize the fault information of real UAV.

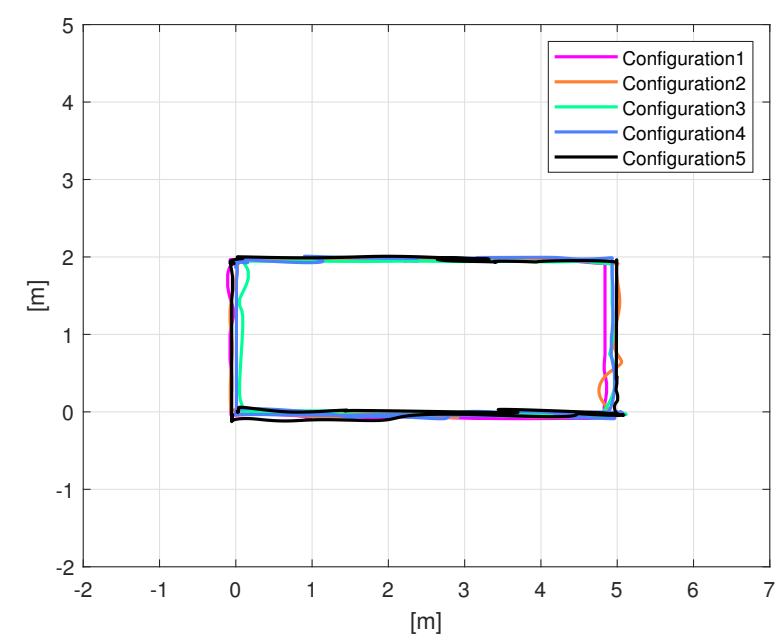

Figure 7. Quadrotor flight trajectory curves under different experimental configurations.

Table 1. Five test configurations.

\begin{tabular}{ccc}
\hline Tests & Propeller Sets & Labels \\
\hline Configuration1 & four propellers in good condition & 0 \\
Configuration2 & The propeller of No.1 motor is damaged & 1 \\
Configuration3 & The propeller of No.2 motor is damaged & 2 \\
Configuration4 & The propeller of No.3 motor is damaged & 3 \\
Configuration5 & The propeller of No.4 motor is damaged & 4 \\
\hline
\end{tabular}

In order to ensure the diversity of collected data, this paper conducted $10 \mathrm{flight}$ experiments for each group based on the above five propellers configuration modes, finally collected about 500,000 pieces of real flight data and labeled the experimental data.

\subsection{Data Preparation and Data Augmentation}

After obtaining the original data, remove the first $5 \mathrm{~s}$ and the last $5 \mathrm{~s}$ of the collected data according to the nature of the sensor, so as to eliminate the influence of abnormal data during takeoff and landing. The final intercepted data is used as the input of sample data.

The data of the UAV at a certain time can only represent the instantaneous state of the $\mathrm{UAV}$, not its health state. Therefore, in order to further obtain the sample data, the data in this paper is divided into short data segments. According to the previous experience [33], the length of the divided data segments is set to 80, that is, the step size of each sample segment is 80 , The collected data is expressed as Data $=\left[\left(x_{1}, y_{1}\right), \cdots,\left(x_{i}, y_{i}\right)\right]^{T}$, Data is the segmented data set, $x_{i}$ is the single sample data, each sample contains 80 pieces of sampling data, and $y_{i}$ is the fault category label of the sample data. 
The best way to enhance the generalization ability of deep neural networks is to use more training samples [34]. When dividing sample data, in order to extract more effective features from original sequence data and obtain as many sample data as possible, this paper uses the equally spaced sliding window to overlap sampling data. The calculation formula for realizing overlapping sampling is as follows:

$$
N=\frac{L_{1}-L_{2}}{S}+1
$$

where, $N$ is the number of samples after overlapping sampling; $L_{1}$ is the length of original data; $L_{2}$ is the length of a single sample, namely the width of the window; $S$ is the moving step of the sliding window, namely the sampling interval; in this paper, $L_{2}=80, S=30$.

Finally, in order to unify the data dimension, the data is normalized. Among them, the positive and negative of the attitude-related data represents the direction of the UAV, so the attitude-related data is normalized to the interval $[-1,1]$, and the motor speed data is normalized to the interval $[0,1]$.

\subsection{Model Determination Experiment of Quadrotor Fault Diagnosis Based on 1D-WIDRSN}

In this paper, 1D-WIDRSN quadrotor fault diagnosis algorithm based on progressive semi-soft threshold is constructed. The input of the algorithm is the nine characteristic parameters of the UAV, and the fault type of the UAV is output after calculation.

We uses the Pytorch framework to build the designed 1D-WIDRSN fault diagnosis model in the Python3.8 environment. To verify the effectiveness of the model, a series of experiments are carried out on the computer.

In the training process, the batch-size is 128 , the epochs is 80 , the learning rate of Adam algorithm is 0.001 , and the cross entropy loss function was used as the objective function to conduct model training. The input layer of the model is the data samples mentioned above, and the input size is (None, 80,9). None is the number of input samples, 80 is the time step of the samples, and 9 is the nine characteristic variables of UAV.

How to find suitable parameters for the model is very important. In this paper, a series of experiments are carried out to determine the values of relevant parameters of the model. Each group of experiments is carried out for 5 times, and the average accuracy of 5 experiments is taken as the evaluation standard of the model [35]. The parameters considered in the experiment are: the size of the first wide convolution kernels, the number of the wide convolution kernels, the number of residual modules, and the block size discarded by the DropBlock layer.

The wide convolution kernels can better extract short-time fault features for time series data, thereby enhancing the model's anti-noise performance. For the quadrotor sample data in this paper, the convolution kernels used should be $n \times 9$, which $n$ is the size of the convolution kernels in the time domain, and 9 is the number of feature types. On the issue of convolution kernels selection, a smaller convolution kernels may not be able to fully express the characteristics of the input data, while a larger convolution kernels may increase the complexity of the model, and the number of convolution kernels will also affect the experiment results.

To determine the size of the wide convolution kernels, set the experiment as follows: the number of convolution kernels at the first layer is set to 24 , the size is set to $8,12,24,32$, 48 the number of residual shrinkage modules is set to 1 , and the size of the convolution kernels of the residual shrinkage modules is set to 3 . The final parameter value is determined by analyzing the influence of convolution kernels size on model performance in the wide convolution layer. As show in Table 2, the accuracy increases with the increase of the size of convolution kernels. When the size of wide convolution kernels reaches 32, the accuracy tends to stabilize, so the size of wide convolution kernels is set to 32. 
Table 2. Influence of the size of convolution kernels in the wide convolution layer on 1DWIDRSN model.

\begin{tabular}{cccccc}
\hline The Size of the Wide Convolution Kernels & $\mathbf{8}$ & $\mathbf{1 2}$ & $\mathbf{2 4}$ & $\mathbf{3 2}$ & $\mathbf{4 8}$ \\
\hline Accuracy $(\%)$ & 87.46 & 90.39 & 92.33 & 93.81 & 93.86 \\
\hline
\end{tabular}

After determining the size of the convolution kernels of the wide convolution layer, further experiments are carried out to select the number of wide convolution kernels. The size of the wide convolution kernels is set to 32 , and the number is set to $16,24,32,48$, and 64. As show in Table 3, the accuracy increases with the number of convolution kernels. When the number of wide convolution kernels reaches 32, the accuracy tends to stabilize, so the number of wide convolution kernels is set to 32 .

Table 3. Influence of the number of convolution kernels in the wide convolution layer on 1DWIDRSN model.

\begin{tabular}{cccccc}
\hline The Number of the Wide Convolution Kernels & $\mathbf{1 6}$ & $\mathbf{2 4}$ & $\mathbf{3 2}$ & $\mathbf{4 8}$ & $\mathbf{6 4}$ \\
\hline Accuracy $(\%)$ & 92.73 & 93.97 & 94.83 & 94.62 & 95.17 \\
\hline
\end{tabular}

Further experiments were carried out on the number of residual shrinkage modules (RSMs), and the number of RSMs are set to 1, 2, 3, 4, 5. As show in Table 4, when the number of RBUs is greater than 4 , the accuracy becomes stable. However, the increase in the number of RSMs will reduce the network training speed, so the number of RSMs in this model is set to 3 .

Table 4. Influence of the number of residual shrinkage modules on 1D-WIDRSN model.

\begin{tabular}{cccccc}
\hline The Number of Residual Shrinkage Modules & $\mathbf{1}$ & $\mathbf{2}$ & $\mathbf{3}$ & $\mathbf{4}$ & $\mathbf{5}$ \\
\hline Accuracy (\%) & 94.50 & 96.13 & 97.95 & 98.12 & 98.53 \\
\hline
\end{tabular}

Adding DropBlock layer after wide convolution layer, we need to set the block size in DropBlock. As show in Table 5, the model has the highest accuracy when the block size is 5, so the size of block size in DropBlock is set to 5 .

Table 5. Influence of block size in the DropBlock layer on 1D-WIDRSN model.

\begin{tabular}{ccccc}
\hline The Block Size of the DropBlock & $\mathbf{1}$ & $\mathbf{3}$ & $\mathbf{5}$ & $\mathbf{7}$ \\
\hline Accuracy (\%) & 98.18 & 98.36 & 98.24 & 98.32 \\
\hline
\end{tabular}

In the network model designed in this paper, the input sample will first pass through a wide convolution layer. The convolution kernels size of the wide convolution layer is $32 \times 9$, and the number of convolution kernels is 32. After the wide convolution layer, the DroopBlock technology is adopted to avoid overfitting problem. After the ReLu processing, it enters three residual shrinkage modules. Each residual shrinkage module has two convolution layers. In order to increase the receptive field and reduce parameters at the same time, the convolution kernels size of each convolution layer is 3 , and the number of convolution kernels in each module is twice that of the previous module. After the average pooling layer processing, the output features are expanded into one-dimensional vectors and are input into the full connection layer. Finally, the fault diagnosis results are obtained through the Softmax layer. The five fault types correspond to the five outputs of the Softmax layer, respectively.

The specific structural parameters of the quadrotor fault diagnosis model are shown in Table 6. 
Table 6. The network parameters of the 1D-WIDRSN model.

\begin{tabular}{ccc}
\hline Layer Type & Windows Size & Kernel Number \\
\hline Input & - & - \\
Conv1D & 16 & 32 \\
$\mathrm{RSM}_{1}$ Conv $_{1}$ & 3 & 64 \\
$\mathrm{RSM}_{1}$ Conv $_{2}$ & 3 & 64 \\
$\mathrm{RSM}_{2}$ Conv $_{1}$ & 3 & 128 \\
$\mathrm{RSM}_{2 \_C o n v_{2}}$ & 3 & 128 \\
$\mathrm{RSM}_{3 \_C o n v_{1}}$ & 3 & 256 \\
$\mathrm{RSM}_{3 \_C o n v_{2}}$ & 3 & 256 \\
AvgPool1D $_{\text {Fully Connected }}$ & - & - \\
Softmax & - & - \\
\hline
\end{tabular}

\subsection{Experiment Results}

After determining the model parameters, input the data samples into the fault diagnosis model. The training process is visualized as shown in Figure 8, which records the curve of loss and accuracy of training set and test set. After about 20 iterations, the accuracy rate of the model gradually stabilizes and maintains at about $98 \%$.
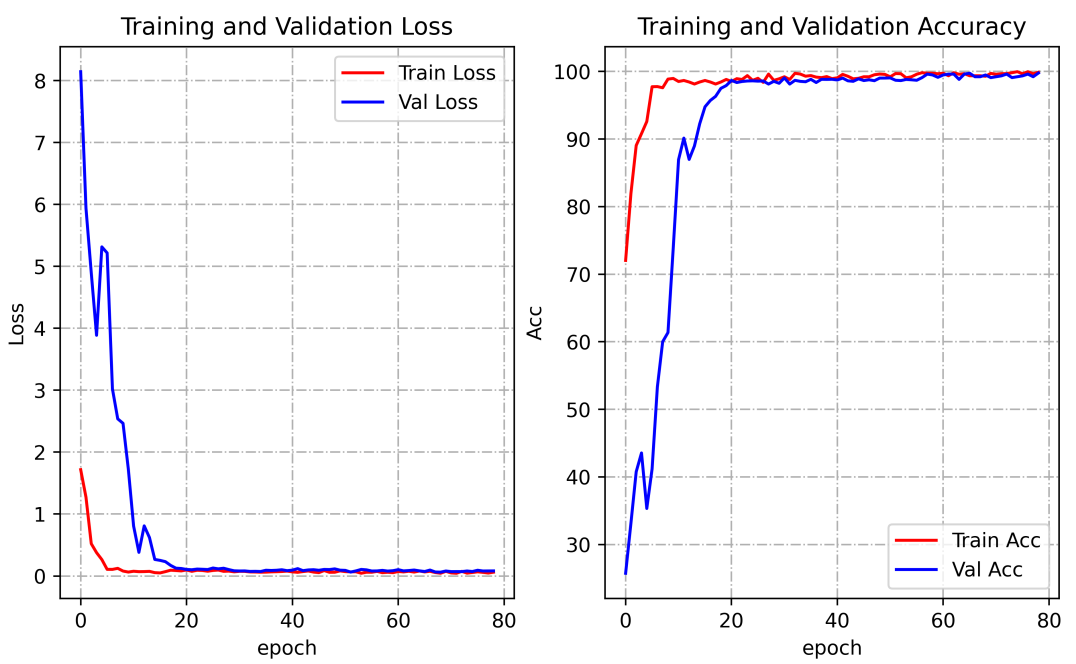

Figure 8. Loss and accuracy curves of training set and test set.

\section{Evaluation and Discussion}

\subsection{Evaluation of Model Feature Extraction Capability}

In order to verify the feature extraction ability of the algorithm, $t$-distributed stochastic neighbor embedding ( $\mathrm{t}$-SNE) [36] can be used to visually analyze the original flight data and the data after model calculation. T-SNE is a popular dimensionality reduction algorithm. This paper uses the t-SNE algorithm to map the data to a two-dimensional scatter graph to evaluate the effectiveness of features. The visualization results are shown in Figure 9. It can be seen that most of the failure categories of the unprocessed raw flight data are overlapping and disorderly. After model feature extraction, the four categories can be almost completely separated. Therefore, the model has strong ability of fault feature extraction. 


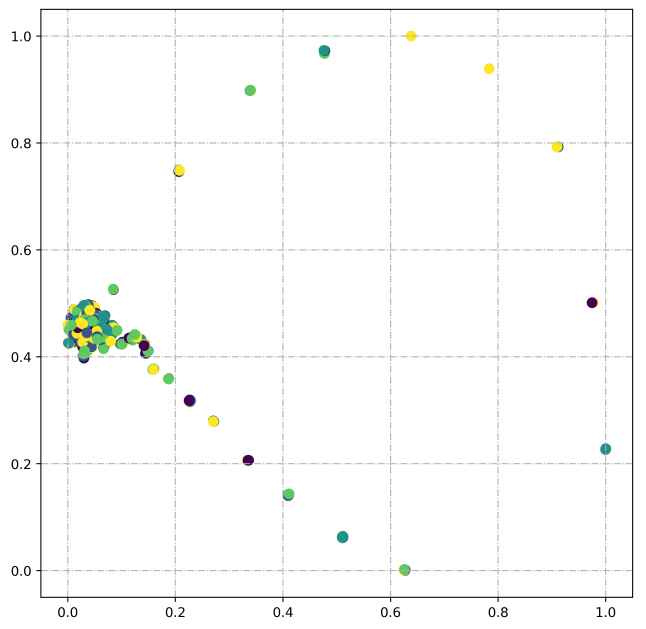

(a)

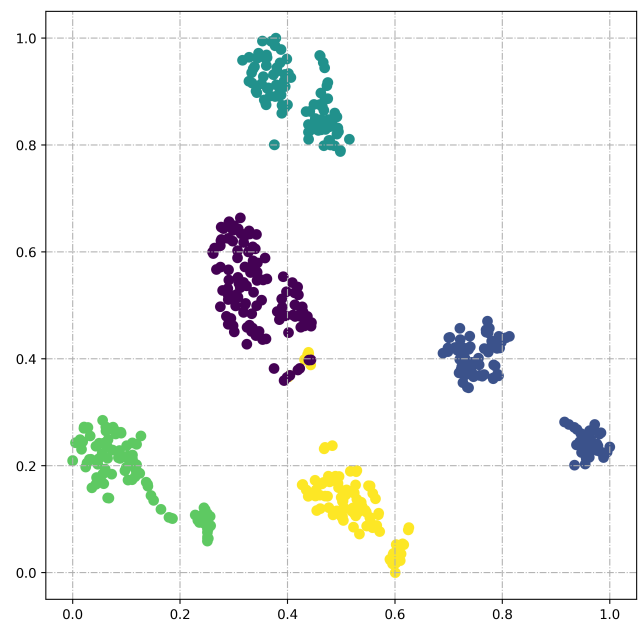

(b)

Figure 9. Visualization of the feature distribution via t-SNE method: (a) Visualization of raw flight data; and (b) visualization of features extracted by the 1D-WIDRSN model.

\subsection{Diagnostic Performance of Different Models}

In order to verify the effectiveness of the algorithm, this paper compares 1D-WIDRSN method with other fault diagnosis methods. In the comparative experiment, DNN, CNN, Vgg [37], Resnet, 1D-WDRSN are selected, respectively. As show in Table 7, compared with other fault diagnosis models, 1D-WIDRSN has higher accuracy.

Then, this section uses the Confusion Matrix to measure the accuracy of the fault diagnosis model. The Confusion Matrix can evaluate the performance of the classification model by counting the number of correct and wrong classifications. Figure 10 is the confusion matrix of different fault diagnosis models, in which the abscissa is the diagnosis result of the diagnosis model and the ordinate is the actual fault category label. As show in Figure 10, compared with other fault diagnosis models, 1D-WIDRSN can identify various faults more effectively.

Table 7. Comparison of accuracy results of other models.

\begin{tabular}{cc}
\hline The Name of the Model & Accuracy (\%) \\
\hline DNN & 86.71 \\
CNN & 91.80 \\
Vgg & 94.92 \\
ResNet & 97.07 \\
1D-WDRSN & 97.85 \\
1D-WIDRSN & 98.44 \\
\hline
\end{tabular}

From the above results, it can be concluded that compared with other comparison methods, the 1D-WIDRSN proposed in this paper has a better effect. The reason can be explained as follows: Firstly, the residual shrinkage module of 1D-WIDRSN uses the induction mechanism to find the interference information of the input sample, and uses the progressive semi-soft threshold function to set the interference information to zero, so as to reduce the influence of the interference information on the fault diagnosis accuracy. Secondly, the wide convolutional layer of the 1D-WIDRSN model can effectively extract the short-term features of the original data, which helps the model find the key information of different fault categories. Finally, the DroupBlock algorithm is introduced to avoid the dependence of the model on specific neurons and prevent the model from overfitting by randomly discarding some characteristic elements. 


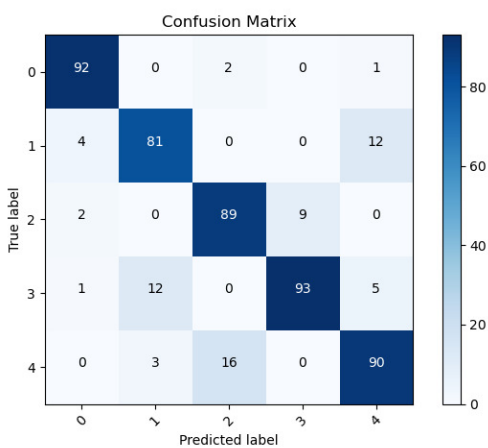

(a)

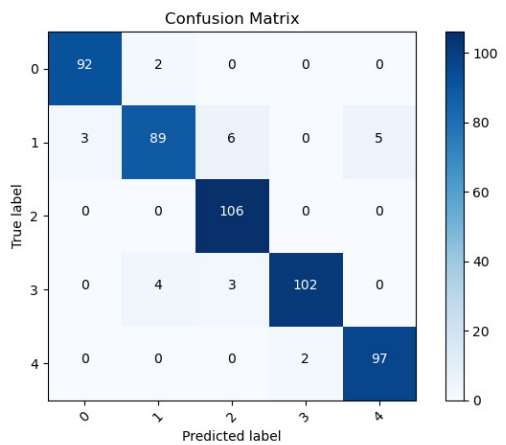

(c)

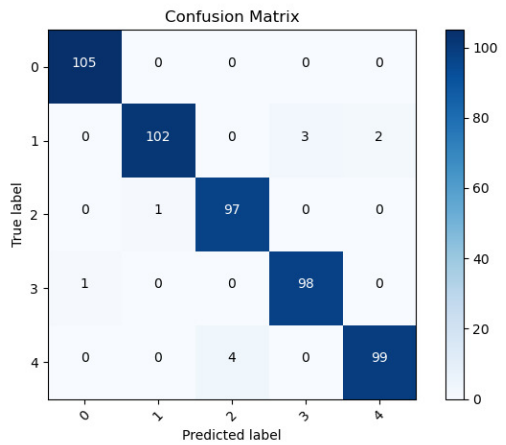

(e)

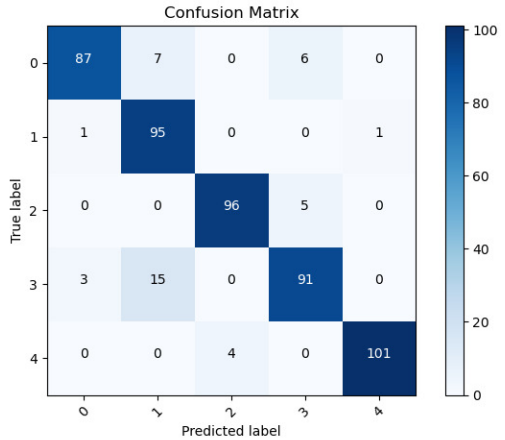

(b)

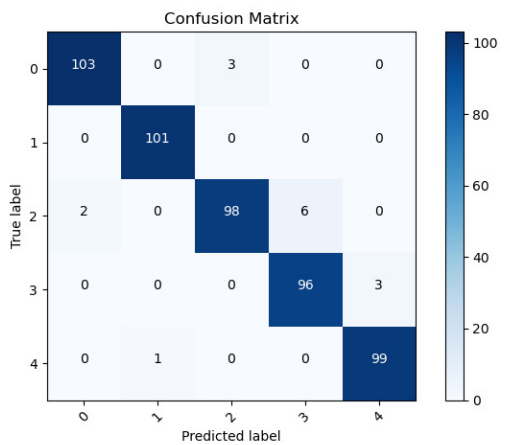

(d)

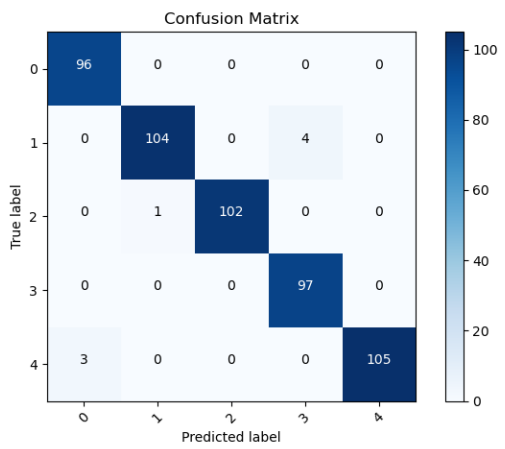

(f)

Figure 10. Confusion Matrix of different fault diagnosis models: (a) DNN; (b) CNN; (c) Vgg; (d) ResNet; (e) 1D-WDRSN; and (f) 1D-WIDRSN.

\section{Conclusions}

In engineering applications, inevitable environmental interference information and UAV own fault-tolerant control will seriously affect the accuracy of fault diagnosis when the early fault of quadrotor actuator occurs. We proposed 1D-WIDRSN fault diagnosis algorithm, which can adaptively extract essential features from the raw flight data, developed to realize end-to-end fault diagnosis. This diagnostic algorithm is used in the flight-test inspection of quadrotor before or after use.

1D-WIDRSN replaces soft threshold in DRSN with progressive semi-soft threshold function, which preserves as many effective features as possible in the original data. As the shrinking layer of residual shrinkage module, the progressive semi-soft threshold can reduce the influence of interference information in the original data on fault diagnosis. In addition, the wide convolution layer can better extract the short-time features of faults and further reduce the influence of interference information. The DroupBlock layer uses the method of randomly discarding feature blocks to suppress over-fitting of the model. 
Finally, a certain type of quadrotor is selected for the experiment, and the speed, angular velocity, and angular acceleration are used as the experimental data to complete the training and testing of the model, so as to realize the early failure detection and location of the actuator of the quadrotor. Experimental results show that the fault diagnosis method based on 1D-WIDRSN can effectively identify the quadrotor propeller faults under interference, and has good sensitivity in the early stage of minor faults. Compared with other fault diagnosis methods, this method has better performance. Therefore, the quadrotor fault diagnosis based on 1D-WIDRSN is a good solution for the field of UAV health detection.

In the future research, we will focus on the compound fault diagnosis of quadrotor aircraft to improve the practicality of the 1D-WDRSN fault diagnosis algorithm. Some issues concerning the implementation of the 1D-WDRSN algorithm in the onboard controller of UAV should also be addressed to improve the effectiveness of the algorithm in real-time fault detection during flight.

Author Contributions: All authors designed this work; H.G. and P.Y. prepared an initial draft and overall structure of this research paper and invited other authors to contribute and expand the research scope; P.L. and C.W. supervised the research and edited the manuscript. All authors have read and agreed to the published version of the manuscript.

Funding: Partial support of this research was provided by the National Science Key Lab Fund Project (6142605200402), the Aeronautical Science Foundation of China (20200007018001), the National Natural Science Foundation of China (61922042), the Aero Engine Corporation of China Industryuniversity-research cooperation project (HFZL2020CXY011) and the Research Fund of State Key Laboratory of Mechanics and Control of Mechanical Structures (MCMS-I-0121G03). Any opinions, findings, and conclusions, or recommendations expressed in this material are those of the authors and do not necessarily reflect the views of the sponsoring agency.

Institutional Review Board Statement: Not applicable.

Informed Consent Statement: Not applicable.

Data Availability Statement: Data sharing not applicable due to privacy and ethical restrictions.

Conflicts of Interest: The authors declare no conflict of interest.

\section{References}

1. Dong, H. K.; Huh, J.H.; Kim, J.D. Design and Implementation of Drone for Wideband Communication and Long-range in Maritime. In Information Science and Applications (ICISA); Springer: Singapore, 2016.

2. Valente, J.; Cerro, J.D.; Barrientos, A.; Sanz, D. Aerial coverage optimization in precision agriculture management: A musical harmony inspired approach. Comput. Electron. Agric. 2013, 99, 153-159. [CrossRef]

3. Tziavou, O.; Pytharouli, S.; Souter, J. Unmanned Aerial Vehicle (UAV) based mapping in engineering geological surveys. Consid. Optim. Results 2018, 232, 12-21.

4. Barmpounakis, E.N.; Vlahogianni, E.I.; Golias, J.C. Unmanned Aerial Aircraft Systems for transportation engineering: Current practice and future challenges. Int. J. Transp. Sci. Technol. 2017, 5, 111-122. [CrossRef]

5. Chang, D.E.; Eun, Y. Global Chartwise Feedback Linearization of the Quadcopter With a Thrust Positivity Preserving Dynamic Extension. IEEE Trans. Autom. Control 2017, 62, 4747-4752. [CrossRef]

6. Zhang, H.; Gao, Q.; Pan, F. An Online Fault Diagnosis Method for Actuators of Quadrotor UAV with Novel Configuration Based on IMM. In Proceedings of the Chinese Automation Congress (CAC), Shanghai, China, 6-8 November 2020.

7. Avram, R.C.; Zhang, X.; Muse, J. Quadrotor actuator fault diagnosis and accommodation using nonlinear adaptive estimators. IEEE Trans. Control Syst. Technol. 2017, 25, 2219-2226. [CrossRef]

8. Bauer, P.; Venkataraman, R.; Vanek, B.; Seiler, P.J.; Bokor, J. Fault Detection and Basic In-Flight Reconfiguration of a Small UAV Equipped with Elevons. IFAC-PapersOnLine 2018, 51, 600-607. [CrossRef]

9. Miao, Q.; Wei, J.; Wang, J.; Chen, Y. Fault Diagnosis Algorithm Based on Adjustable Nonlinear PI State Observer and Its Application in UAV Fault Diagnosis. Algorithms 2021, 14, 119. [CrossRef]

10. Kim, T.; Kim, S. Actuator fault diagnosis and counterplan using extended kalman filter considering symmetry of hexacopter UAV. J. Inst. Control. Robot. Syst. 2021, 27, 473-481. [CrossRef]

11. Antonio G.R.; Agus H.; Poramate M. Robust Actuator Fault Diagnosis Algorithm for Autonomous Hexacopter UAVs. IFACPapersOnLine 22020, 53, 682-687. 
12. De Jesus Rangel-Magdaleno, J.; Ureña-Ureña, J.; Hernández, A.; Perez-Rubio, C. Detection of unbalanced propeller on UAV by means of audio signal. In Proceedings of the IEEE International Autumn Meeting on Power, Electronics and Computing, Ixtapa, Mexico, 14-16 November 2018.

13. Yousefi, P.; Fekriazgomi, H.; Demir, M.A.; Prevost, J.J.; Jamshidi, M. Data-Driven Fault Detection of Un-Manned Aerial Vehicles Using Supervised Learning Over Cloud Networks. In Proceedings of the World Automation Congress, Stevenson, WA, USA, 3-6 June 2018.

14. Park, J.H.; Jun, C.Y.; Jeong, J.Y.; Chang, D.E. Real-time quadrotor actuator fault detection and isolation using multivariate statistical analysis techniques with sensor measurements. In Proceedings of the 20th International Conference on Control, Automation and Systems, Busan-City, Korea, 13-16 October 2020.

15. Bondyra, A.; Gasior, P.; Gardecki, S.; Kasiński, A. Fault diagnosis and condition monitoring of uav rotor using signal processing. In Proceedings of the Signal Processing: Algorithms, Architectures, Arrangements, and Applications, Poznan, Poland, 19-21 September 2018.

16. Altinors, A.; Yol, F.; Yaman, O. A sound based method for fault detection with statistical feature extraction in UAV motors. Appl. Acoust. 2021, 183, 108325. [CrossRef]

17. Chen, Y.; Zhang, C.; Zhang, Q.; Hu, X. UAV fault detection based on GA-BP neural network. In Proceedings of the 32nd Youth Academic Annual Conference of Chinese Association of Automation, Hefei, China, 19-21 May 2017.

18. Guo, D.; Zhong, M.; Ji, H.; Liu, Y.; Yang, R. A hybrid feature model and deep learning based fault diagnosis for unmanned aerial vehicle sensors. Neurocomputing 2018, 319, 155-163. [CrossRef]

19. Iannace, G.; Ciaburro, G.; Trematerra, A. Fault Diagnosis for UAV Blades Using Artificial Neural Network. Robotics 2019, 8, 59. [CrossRef]

20. Gao, T.; Sheng, W.; Zhou, M.; Fang, B.; Zheng, L. MEMS Inertial Sensor Fault Diagnosis Using a CNN-Based Data-Driven Method. Int. J. Pattern Recognit. Artif. Intell. 2020, 34, 2059048. [CrossRef]

21. Xiao, Q.X. A Sensor Fault Diagnosis Algorithm for UAV Based on Neural Network. In Proceedings of the 2021 International Conference on Intelligent Transportation, Indianapolis, IN, USA, 19-22 September 2021.

22. Liu, W.; Chen, Z.; Zheng, M. An Audio-Based Fault Diagnosis Method for Quadrotors Using Convolutional Neural Network and Transfer Learning. In Proceedings of the American Control Conference, New Orleans, LA, USA, 25-28 May 2021.

23. Jia, Z.; Liu, Z.B.; Cai, Y.Y. A novel fault diagnosis method for aircraft actuator based on ensemble model. Measurement 2021, 176, 109235. [CrossRef]

24. Chen, H.; Ye, W. Classification of human activity based on radar signal using 1-D convolutional neural network. IEEE Geosci. Remote Sens. Lett. 2019, 17, 1178-1182. [CrossRef]

25. Guo, Q.; Li, Y.; Song, Y.; Wang, D.; Chen, W. Intelligent fault diagnosis method based on full 1-D convolutional generative adversarial network. IEEE Trans. Ind. Inform. 2019, 16, 2044-2053. [CrossRef]

26. He, K.; Zhang, X.; Ren, S.; Sun, J. Deep residual learning for image recognition. In Proceedings of the IEEE Conference on Computer Vision and Pattern Recognition, Las Vegas, NV, USA, 27-30 June 2016.

27. Zhao, M.; Zhong, S.; Fu, X.; Tang, B.; Pecht, M. Deep residual shrinkage networks for fault diagnosis. IEEE Trans. Ind. Inform. 2020, 16, 4681-4690. [CrossRef]

28. Zhang, Z.; Li, H.; Chen, L.; Han, P. Rolling bearing fault diagnosis using improved deep residual shrinkage networks. Shock Vib. 2021, 2021, 1-11.

29. Lei, S.; Lu, M.; Lin, J.; Zhou, X.; Yang, X. Remote sensing image denoising based on improved semi-soft threshold. Signal. Image Video Process. 2021, 15, 73-81. [CrossRef]

30. Zhang, W.; Peng, G.; Li, C.; Chen, Y.; Zhang, Z. A new deep learning model for fault diagnosis with good anti-noise and domain adaptation ability on raw vibration signals. Sensors 2017, 17, 425. [CrossRef] [PubMed]

31. Xiang, C.; Ren, Z. An Intelligent Fault Diagnosis Method of Rolling Bearing with Wide Convolution Kernel Network. In Proceedings of the International Conference on Automation, Control and Robotics Engineering, Dalian, China, 18-20 September 2020.

32. Ghiasi, G.; Lin, T.Y.; Le, Q.V. Dropblock: A regularization method for convolutional networks. arXiv 2018, arXiv:1810.12890.

33. Fu, J.; Sun, C.; Yu, Z.; Liu, L. A hybrid CNN-LSTM model based actuator fault diagnosis for s UAVs. In Proceedings of the Chinese Control Furthermore, Decision Conference, Nanchang, China, 3-5 June 2019.

34. Song, X.; Cong, Y.; Song, Y.; Chen, Y.; Liang, P. A bearing fault diagnosis model based on CNN with wide convolution kernels. J. Ambient. Intell. Humaniz. Comput. 2021, 1-16. [CrossRef]

35. Yang, J.; Gao, T.; Jiang, S.; Li, S.; Tang, Q. Fault diagnosis of rotating machinery based on one-dimensional deep residual shrinkage network with a wide convolution layer. Shock Vib. 2020, 4, 1-12.

36. Van der Maaten, L.; Hinton, G. Visualizing data using t-SNE. J. Mach. Learn. Res. 2008, 9, 2579-2605.

37. Simonyan, K.; Zisserman, A. Very deep convolutional networks for large-scale image recognition. arXiv 2014, arXiv:1409.1556. 